\title{
Phospholipid Analysis by Nanoflow Liquid Chromatography-Tandem Mass Spectrometry
}

\author{
Myeong Hee Moon* \\ Department of Chemistry, Yonsei University, Seoul, 120-749, South Korea
}

Received February 5, 2014; Revised February 25, 2014; Accepted February 25, 2014

First published on the web March 30, 2014; DOI: 10.5478/MSL.2014.5.1.1

1. Introduction

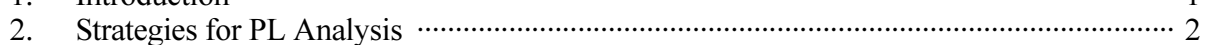

2.1. Extraction of PLs

2.2. nLC-ESI-MS/MS set-up

2.3. Separation and characterization of PLs by nLC-ESI-MS/MS

2.4. LiPilot: a computer software package for PL identification

2.5. Effect of ionization modifiers on the ESI of PLs

2.6. Quantitative analysis of PLs by nLC-ESI-MS/MS

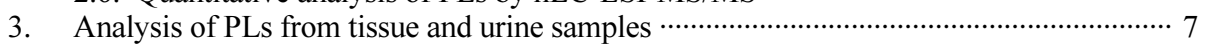

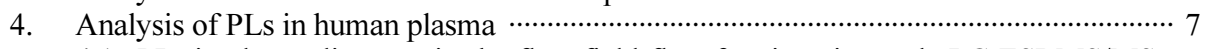

4.1. PLs in plasma lipoproteins by flow field-flow fractionation and nLC-ESI-MS/MS

4.2. Characterization of oxidized PLs in oxidized LDLs

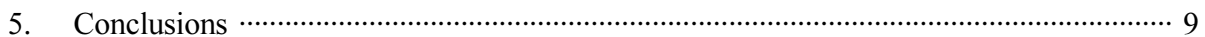

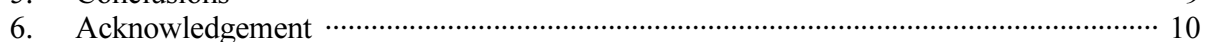

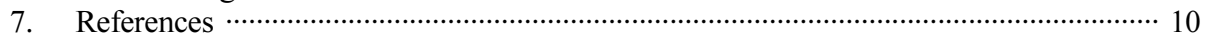

\begin{abstract}
Lipids play important roles in biological systems; they store energy, play a structural role in the cell membrane, and are involved in cell growth, signal transduction, and apoptosis. Phospholipids (PLs) in particular have received attention in the medical and lipidomics research fields because of their involvement in human diseases such as diabetes, obesity, atherosclerosis, and many cancers associated with lipid metabolic disorders. Here I review experimental strategies for PL analysis based on nanoflow liquid chromatography-electrospray ionization-tandem mass spectrometry (nLC-ESI-MS ${ }^{\mathrm{n}}$ ). In particular, discussed are lipid extraction methods, nanoflow LC separation of PLs, effect of ionization modifiers on the ESI of PLs, influence of chain lengths and unsaturation degree of acyl chains of PLs on MS intensity, structural determination of the molecular structure of PLs and their oxidized products, and quantitative profiling of PLs from biological samples such as tissue, urine, and plasma in relation to cancer and coronary artery disease.
\end{abstract}

Keywords: lipid, phospholipid, nLC-ESI-MS/MS, lipidomics, oxidized PLs

\section{Introduction}

Lipidomics is a rapidly growing research area with the major research goals of understanding lipid metabolism and developing biomarkers for human diseases by studying

\section{Open Access}

*Reprint requests to Myeong Hee Moon

E-mail: mhmoon@yonsei.ac.kr

All MS Letters content is Open Access, meaning it is accessible online to everyone, without fee and authors' permission. All MS Letters content is published and distributed under the terms of the Creative Commons Attribution License (http://creativecommons.org /licenses/by/3.0/). Under this license, authors reserve the copyright for their content; however, they permit anyone to unrestrictedly use, distribute, and reproduce the content in any medium as far as the original authors and source are cited. For any reuse, redistribution, or reproduction of a work, users must clarify the license terms under which the work was produced. disease-related changes in the compositions of various lipids. ${ }^{1}$ Lipids are water-insoluble molecules with a variety of polarities and are essential components of cell membranes; phospholipids (PLs) in particular are the major components of biological membranes. PLs play crucial roles in cellular systems including energy storage, signal transduction between cells, cell growth, proliferation, and apoptosis. ${ }^{1,2,3}$ Recent reports have demonstrated that PLs are potential biomarkers of a number of adult diseases including breast cancer, ${ }^{1,2,3,4}$ ovarian cancer, ${ }^{1,2}$ colorectal cancer, ${ }^{11}$ prostate cancer, ${ }^{12,13}$ and coronary artery disease (CAD). ${ }^{14}$

PLs contain a polar head group along with one or two acyl chains attached to glycerol. Their compositions are diverse due to diversity in polar head groups, different lengths (C8 C24) of the acyl chain, and variations in the degree of unsaturation, as shown in Figure 1. PLs with only one acyl chain at either the 


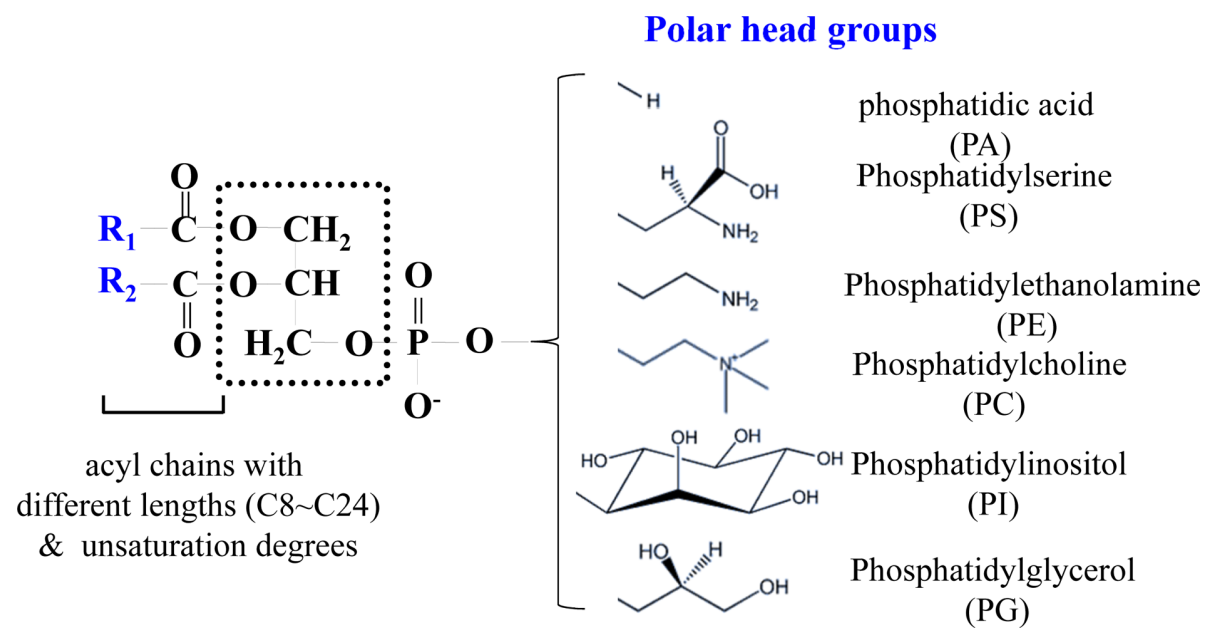

Figure 1. Molecular structure of phospholipids with six different polar head groups.

sn-1 or sn-2 position are referred to as lysophospholipids (LPLs). PLs are named after the head group, e.g. phosphatidylcholine (PC), phosphatidylethanolamine (PE), phosphatidylglycerol (PG), phosphatidylinositol (PI), phosphatidylserine (PS), and phosphatidic acid (PA). Analysis of intact PLs without prior derivatization has been carried out with thin layer chromatography (TLC) using densitometry ${ }^{15,16}$ or matrix-assisted laser desorption time-of-flight mass spectrometry (MALDI-TOF-MS). ${ }^{17,18}$ Rapid advances in MS technology have led to sophisticated detection of PLs using electrospray ionization (ESI). While ESI-MS alone allows high speed analysis of lipid molecules, ${ }^{4,19,20,21}$ lipids with complicated structures and ion suppression from high abundant species are issues even for high-resolution MS. Coupling liquid chromatography (LC) to ESI-MS/MS ${ }^{20,22,23}$ has facilitated the structural determination of lipid species from complicated mixtures, enabling generation of extensive libraries of lipids. Recently, nanoflow LC-ESI-MS/MS has been shown to be feasible for qualitative and quantitative analysis of PLs from various biological samples such as tissue, urine, and plasma. ${ }^{8,24,25,26,27}$

This review deals with the practical use of nanoflow LCESI-MS/MS for the characterization of various PLs from biological samples. Discussed topics are extraction of PLs from biological samples, preparation of capillary columns and $\mathrm{nLC}$ separation, effects of ionization modifiers on PL detection during ESI, utility of LiPilot, which is a computer algorithm for structural determination of PLs, quantitative analysis of PLs, and characterization of urinary PLs from breast cancer and prostate cancer patients along with plasma PLs from CAD patients.

\section{Strategies for PL Analysis}

\subsection{Extraction of PLs}

Due to variations in the electrical propensities of lipids, it is often difficult to extract all lipids at once with high efficiency. PLs have been extracted using the Folch method, ${ }^{28}$ which employs a mixture of chloroform and methanol $(2: 1, \mathrm{v} / \mathrm{v})$, or with the Bligh and Dyer method, ${ }^{29}$ which is based on a reduced solvent to sample ratio compared to the Folch method. In addition to the toxic use of chloroform, the Folch method has often been criticized because it provides relatively low yields of some lysophospholipids. Folch method has been modified using methyl-tert-butyl ether (MTBE) and $\mathrm{CH}_{3} \mathrm{OH} \cdot{ }^{27}$ Extraction using modification of the Folch method was evaluated by comparing the recovery values for six PLs and six LPL standards with different head groups that were spiked into a human plasma sample. Recovery value was calculated from the measured peak area (relative to that of the internal standard added to the plasma sample) of individual species from nLC-ESI-MS before and after extraction using four different extraction methods: Folch with chloroform, Folch with MTBE, $\mathrm{CH}_{3} \mathrm{OH}$ only, and Folch with MTBE/ $\mathrm{CH}_{3} \mathrm{OH}^{27}$ While conventional Folch extraction with chloroform resulted in $58.5 \sim 100 \%$ recovery for 12 species and the method using only methanol resulted in $67.3 \sim 100 \%$ recovery, the modified Folch method with MTBE/ $/ \mathrm{CH}_{3} \mathrm{OH}$ exhibited much higher recovery, mostly above $95 \%$, except for LPC and PE which had recovery values of $84.1 \%$ and $72.1 \%$, respectively. Four extraction methods were used to identify plasma PLs using data-dependent nLC-ESI-MS/ MS analysis. The modified Folch method with MTBE/ $\mathrm{CH}_{3} \mathrm{OH}$ resulted in identification of a total of $120 \mathrm{PLs}$ and LPLs, while only 94 104 PLs and LPLs were identified using the other three extraction methods. Details of extraction using the modified Folch method with MTBE/ $\mathrm{CH}_{3} \mathrm{OH}$ are as follows. For typical extraction of plasma samples, $50 \mu \mathrm{L}$ of plasma is placed in a $2-\mathrm{mL}$ centrifuge tube and diluted to $1 \mathrm{~mL}$ with $0.1 \mathrm{M}$ Tris buffered saline (TBS) solution (adjusted to $\mathrm{pH} 7.4$ by $\mathrm{HCl}$ ) and dried in a vacuum centrifuge for over 6 hours. During drying, an important tip to prevent the loss of PLs due to evaporation 
along with water is to wrap the top of the centrifuge tube without the cap with MilliWrap, a PTFE membrane with $0.45 \mathrm{~mm}$ pores, made by Millipore (Billerica, MA, USA). After drying, $300 \mu \mathrm{L}$ of $\mathrm{CH}_{3} \mathrm{OH}$ is added to the dried powder and after vortexing the mixture, it is placed in an ice bath for 10 minutes. One $\mathrm{mL}$ of MTBE is then added, the mixture is vortexed for 1 hour, and then $250 \mu \mathrm{L} \mathrm{MS}$ grade water is added, followed by an additional hour of vortexing at room temperature. After centrifugation at $1,000 \mathrm{~g}$ for 10 minutes to induce phase separation, the upper organic layer is transferred to a different centrifuge tube. The lower aqueous layer is mixed with $300 \mu \mathrm{L}$ of $\mathrm{CH}_{3} \mathrm{OH}$ for $10 \mathrm{~min}$, sonicated with a tip for $2 \mathrm{~min}$, and centrifuged to collect the supernatant. The supernatant is combined with the previous organic layer and dried in a vacuum centrifuge. Final dried lipids are dissolved in $80 \mu \mathrm{L}$ of $\mathrm{CHCl}_{3}: \mathrm{CH}_{3} \mathrm{OH}(1: 1, \mathrm{v} / \mathrm{v})$, then diluted in $70 \mu \mathrm{L}$ of $\mathrm{H}_{2} \mathrm{O}: \mathrm{CH}_{3} \mathrm{CN}(9: 1, \mathrm{v} / \mathrm{v})$ and $225 \mu \mathrm{L}$ of $\mathrm{CH}_{3} \mathrm{OH}$ to a final concentration of $1 \mathrm{pmol} / \mu \mathrm{L}$, and kept at $-20^{\circ} \mathrm{C}$ for $\mathrm{nLC}$ -

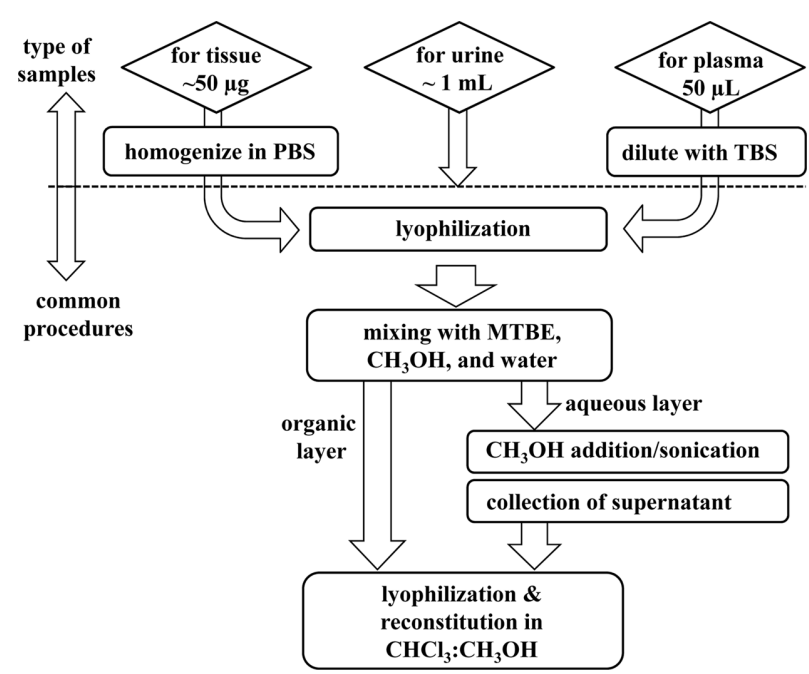

Figure 2. Lipid extraction procedures for LC-ESI-MS ${ }^{\mathrm{n}}$ analysis.
ESI-MS/MS analysis. For urinary lipid extraction, a similar method is utilized; the detailed procedure is described in the

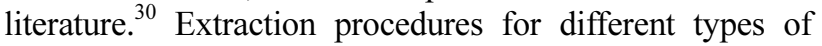
lipid samples are summarized in Figure 2.

\section{2. nLC-ESI-MS/MS set-up}

For nLC-ESI-MS/MS analysis of lipids, a pulled-tip capillary RPLC column without a separate emitter for MS can be simply prepared in the laboratory. First, a fritless capillary column can be made by pulling one end of a fused silica capillary tube $(75 \mu \mathrm{m}$ i.d., $360 \mu \mathrm{m}$ o.d.) while applying a flame to make a cone-shaped emitter end ( $\sim 10 \mu \mathrm{m}$ i.d.). The pulled-tip capillary is then packed with a methanol slurry of reversed-phase $\mathrm{C} 18$ resin beads (100 $\AA$ pore size, $15 \mathrm{~cm}$ for $5 \mu \mathrm{m}$ beads or $7 \mathrm{~cm}$ for $3 \mu \mathrm{m})$ under $\mathrm{He}$ at 1000 psi. Capillary column is connected with a HPLC pump system with a PEEK micro-cross from IDEX Health \& Science (Oak Harbor, WA, USA), in which the other two ends of the micro-cross are connected to Pt wire as an electrical source and to a (split or pressure) capillary tube (20 $\mu \mathrm{m}$ i.d., $360 \mu \mathrm{m}$ o.d.) for splitting pump flow, as shown in Figure 3. The (split or pressure) tube is controlled using a six-port valve. During sample injection, the six-port valve is set-up as shown in Figure 3 so that the split tubing is closed and the lipid sample is loaded onto the capillary column directly from the autosampler at $0.6 \mathrm{~mL} / \mathrm{min}$. For LC separation, gradient flow is delivered from the HPLC pump with the six-port valve using the dotted line configuration so that the pump flow $(7 \sim 10 \mu \mathrm{L} / \mathrm{min})$ is split into the analytical column at $300 \mathrm{~nL} / \mathrm{min}$ while the rest of the flow exits via the pressure tubing. Flow rate of the analytical column is adjusted by varying the length of the pressure tubing (20- $\mu \mathrm{m}$ i.d.). Typical compositions of mobile phase solutions used for gradient elution are $\mathrm{H}_{2} \mathrm{O}: \mathrm{CH}_{3} \mathrm{CN}(90: 10$, v/v) for mobile phase A and isopropanol/ $\mathrm{CH}_{3} \mathrm{CN}(90: 10, \mathrm{v} / \mathrm{v})$ for B. A $0.1 \%$ solution of formic acid is added to these in positive ion mode and $0.05 \%$ ammonium hydroxide is added in negative ion mode to

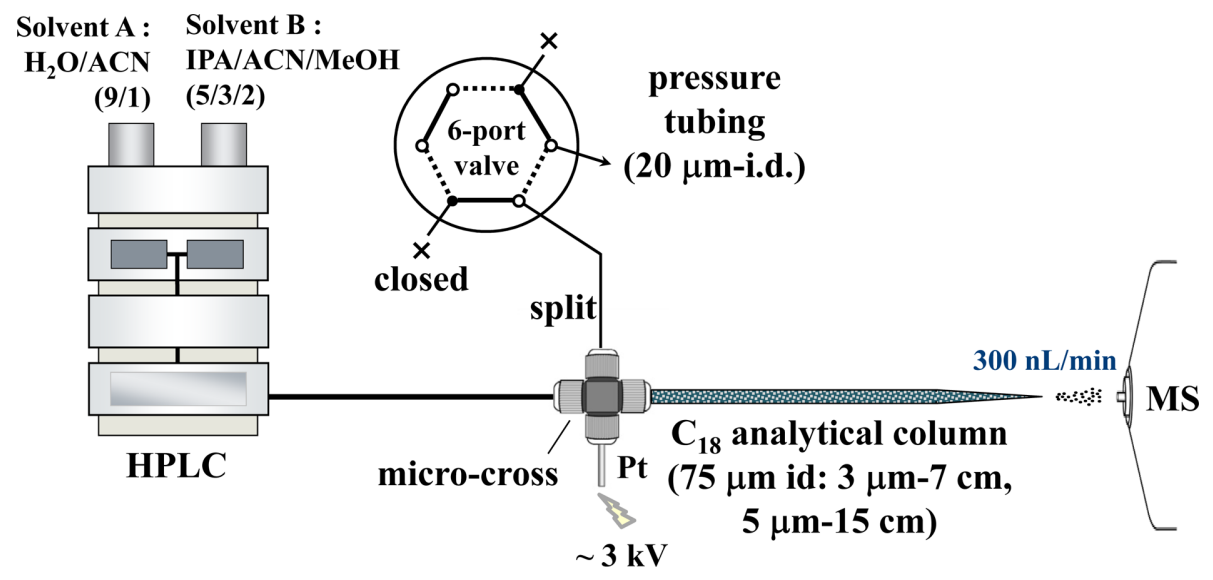

Figure 3. Schematics of nanoflow LC-ESI-MS with a pulled-tip capillary column. 
enhance ionization during ESI. Sample loading is performed using mobile phase $\mathrm{A}$ and gradient elution begins with mobile phase $\mathrm{B}$, which is increased from $0 \%$ to $40 \%$ over $1 \mathrm{~min}$, and then to $100 \%$ over 60 (negative ion mode) $90 \mathrm{~min}$ (positive). For column clean-up and reconditioning, mobile phase $\mathrm{B}$ is maintained at $100 \%$ for $10 \sim 30 \mathrm{~min}$ and then resumed to $0 \%$. ESI voltages are 2.5 (positive) and $3.0 \mathrm{kV}$ (negative). For collision-induced dissociation (CID), data-dependent analysis of the three most intense precursor ions is carried out under $40 \%$ (positive) and $45 \%$ (negative) normalized collision energies. MS ranges suggested for lipid detection are 400-1000 amu for precursor scans and 200-900 amu for MS/MS scans.

\subsection{Separation and characterization of PLs by nLC- ESI-MS/MS}

While MS analysis of most PL classes (especially anionic PLs such as PE, PS, PI, PG, and PA) is performed using the negative ion mode of MS, neutral polar (polar but electrically neutral) PL classes such as PC and sphingomyelin (SM) together with $\mathrm{PE}$ are efficiently detected in positive ion mode. Base peak chromatograms (BPCs) of PL standard mixtures obtained by nLC-ESI-MS/MS in a) positive ion mode and b) negative ion mode along with CID spectra of 12:0/12:0-PC and 18:0/20:4-PI are shown in Figure $4 .^{31}$ Information about the acyl chain structures can be obtained from the CID spectra shown in the inset of Figure 4. As shown in Figure 4a, LPC elutes earlier than PC, and 12:0/ 12:0-PE elutes earlier than PC with the same acyl chain structure, indicating that elution depends on the hydropho- bicity of lipid molecules and the type of head group. Typically, in reversed phase LC, PLs with longer and saturated acyl chains have a longer retention time than those with shorter and unsaturated acyl chains. Molecular structures of lipids can be determined from CID spectra; an example for each ion mode is presented in Figure 3. For the case of PC in Figure 4a, the CID spectrum shows a typical fragment ion at $m / z 523.6\left([\mathrm{M}+\mathrm{H}-183]^{+}\right)$, representing loss of the phosphocholine (183 Da) head group from the parent ion, which is a characteristics of PC. Fragment ions at $\mathrm{m} / \mathrm{z} 479.2$ and 451.2 are due to loss of the acyl chain in the form of carboxylic acid ([M+H-RCOOH $\left.]^{+}\right)$and ions at $\mathrm{m} / z 496.7$ and 468.7 from the loss of the acyl chain in the form of ketene $\left(\left[\mathrm{M}+\mathrm{H}-\mathrm{R}^{\prime} \mathrm{CH}=\mathrm{C}=\mathrm{O}\right]^{+}\right)$. Based on the $\mathrm{CID}$ spectrum, the molecular structure of $\mathrm{PC}$ is $14: 0 / 16: 0-\mathrm{PC}(\mathrm{m} / \mathrm{z} 706.8)$. In Figure $4 \mathrm{~b}$, typical fragments in the form of free carboxylate anions at $m / z 283.3\left(\left[\mathrm{R}_{1} \mathrm{COO}\right]^{-}\right)$and $303.2\left(\left[\mathrm{R}_{2} \mathrm{COO}\right]^{-}\right)$are observed, along with dissociation of the acyl chain from the parent molecule without the head group phosphoinositol (162 Da) at $m / z 438.2$ ([M-H-162- $\left.\left.{ }_{1} \mathrm{COOH}\right]^{-}\right)$and 419.3 ([M$\left.\left.\mathrm{H}-162-\mathrm{R}_{2} \mathrm{COOH}\right]^{-}\right)$in the form of carboxylic acid. Likewise, dissociation of the acyl chain from the parent molecule is clearly observed at $\mathrm{m} / \mathrm{z} 600.7$ and 581.5 in the form of carboxylic acid. CID spectrum in Figure $4 \mathrm{~b}$ allows identification of the phospholipid as 18:0/20:4-PI $(\mathrm{m} / \mathrm{z} 885.4)$.

Figure 4 also shows separation of regioisomers of LPLs based on differences in the hydrophobic interactions of the following LPL standards with the C18 stationary phase: LPC, LPE, LPA, LPG, LPS, and LPI. For instance, peaks 3a and $3 \mathrm{~b}$ in Figure $4 \mathrm{a}$ represent the separation of two
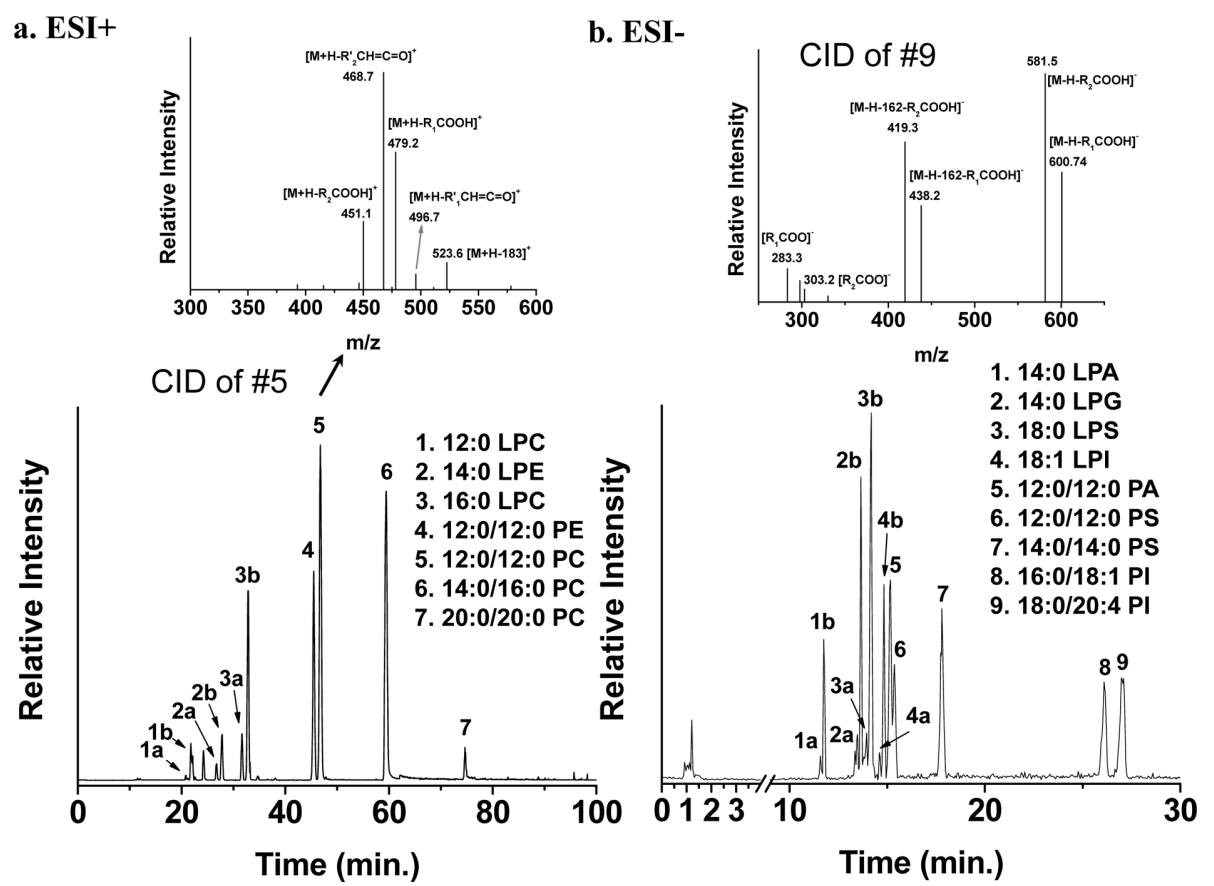

Figure 4. Base peak chromatograms of PL standard mixtures from nLC-ESI-MS/MS in a) positive and b) negative ion mode along with CID spectra of peaks 6 and 9. Permission to reproduce obtained from the publisher of ref. 31 . 


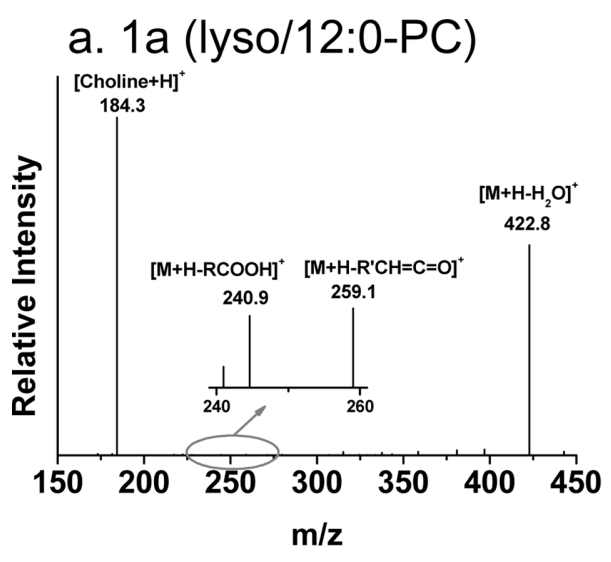

b. $1 b(12: 0 /$ lyso-PC)

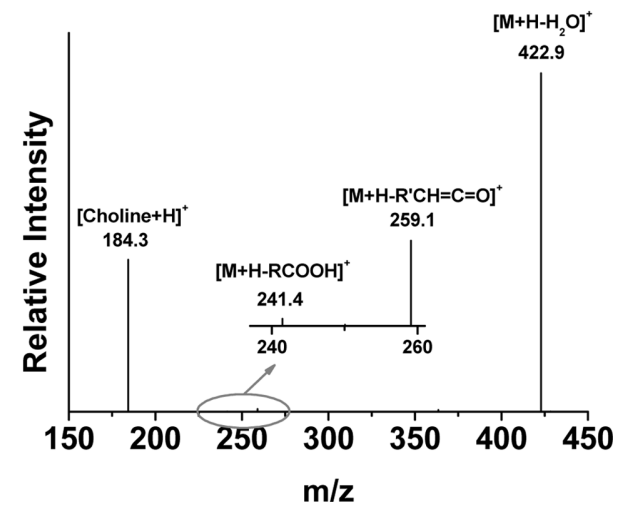

Figure 5. Data-dependent CID spectra of two regioisomers of 12:0-LPC by nLC-ESI-MS/MS. Permission to reproduce obtained from the publisher of ref. 31 .

regioisomers (lyso/16:0-PC and 16:0/lyso-PC). Lyso/16:0PC stands for the hydroxide attached to the sn- 1 carbon of the glycerol backbone, while the acyl chain is located at the sn-2 position. Because there is a difference between the geometrical structures - LPC with the acyl chain located at the sn- 1 position is more extended and therefore interacts more with the stationary phase than LPC with the acyl chain at sn-2- its retention time is longer. Therefore, peak $3 \mathrm{a}$ is from lyso/16:0-PC. Identification of regioisomers can be confirmed not only by the separation time, but also by data-dependent CID experiments during nLC-ESI-MS/MS. An earlier systematic analysis of regioisomers reported that CID spectra of regioisomers showed the same fragment ions, but that the fragment ions had different intensities. ${ }^{31}$ Figure 5 shows two CID spectra of 12:0-LPC regioisomers during $\mathrm{nLC}$-ESI-MS/MS in positive ion mode; there is a difference in the ratio of two prominent fragment ions: $\mathrm{m} / \mathrm{z}$ 184.3 for phosphocholine cation $\left(\mathrm{H}_{2} \mathrm{PO}_{4}\left(\mathrm{CH}_{2}\right)_{2} \mathrm{~N}\left(\mathrm{CH}_{3}\right)_{3}\right.$, $184 \mathrm{amu}$ ) and 422.8 for the parent ion after the loss of water. CID patterns of characteristic fragment ions of regioisomers differ from each other depending on the type of head group; details can be found in ref. 31 .

\subsection{LiPilot: a computer software package for PL identi- fication}

Identification of the molecular structures of PLs often requires a manual examination of CID spectra, because no commercial software is available. Recently, in our laboratory, we developed "LiPilot", a computer-based algorithm for identifying PL molecules, which utilizes entire sets of raw data generated from nLC-ESI-MS/MS experiments, including precursor ion MS scans and datadependent MS/MS spectra. Developed software is flexible in producing and retrieving library files for PLs with different head groups and acyl chain structures that contain customized fragment ion patterns specific to MS instruments, and it compares differences between theoretical and experimental $\mathrm{m} / \mathrm{z}$ values of fragment ions using a novel scoring system called the "confidence score" to maximize identification of molecular structures. This software can differentiate PL and LPL regioisomers and perform filtering. After validating LiPilot by identifying 24 PL standards, we applied our software to a human urinary PL mixture. We successfully identified 115 PLs, which represents a $30 \%$ increase in the number of PLs identified compared to the number identified by manual examination. LiPilot is available to use upon request.

\subsection{Effect of ionization modifiers on the ESI of PLs}

While lipids are often analyzed using two separate nLCESI-MS/MS runs, one in positive ion mode and one in negative ion mode, this approach may not be optimal when a number of samples have to be quantified. There have therefore been efforts to develop an effective ionization modifier for simultaneous analysis of all classes of PLs in negative ion mode to facilitate rapid analysis. Systematic evaluation of ionization efficiencies of 13 different PL classes using a variety of ionization modifiers $(0.05 \%$ $\mathrm{NH}_{4} \mathrm{OH}, 5 \mathrm{mM} \mathrm{NH}_{4} \mathrm{CH}_{3} \mathrm{CO}_{2}, 5 \mathrm{mM} \mathrm{NH}_{4} \mathrm{HCO}_{2}$, and $0.05 \%$ $\mathrm{NH}_{4} \mathrm{OH}+5 \mathrm{mM} \mathrm{NH} \mathrm{HCO}_{2}$ ) revealed that addition of ammonium formate (AF) to ammonium hydroxide (AH) resulted in a significant increase in ionization efficiency of neutral polar lipids to levels similar to those observed for anionic lipids. ${ }^{32}$ Optimization of the concentration of ammonium formate resulted in the selection of $0.05 \% \mathrm{AH}$ plus $1 \mathrm{mM}$ AF for simultaneous separation and detection of 33 PL standards in negative ion mode, as shown in Figure 6 . While the mixed ionization modifier $(0.05 \% \mathrm{AH}$ plus $1 \mathrm{mM}$ AF) allowed simultaneous analysis of 13 different sub-categories of PLs in negative ion mode, it should be noted that PLs with choline head groups, such as PC and $\mathrm{SM}$, were detected as the formate adduct form $\left(\left[\mathrm{M}+\mathrm{HCOO}^{-}\right)\right.$in negative ion mode. $\mathrm{MS}^{2}$ experiments of this precursor ion (formate adduct) predominantly yielded the $\left[\mathrm{M}_{-} \mathrm{CH}_{3}\right]^{-}$ion, therefore $\mathrm{MS}^{3}$ (MS/MS/MS) experiments are required to obtain characteristic fragment ions for structural determination. The developed method was applied to a human urinary sample, and resulted in identification of 


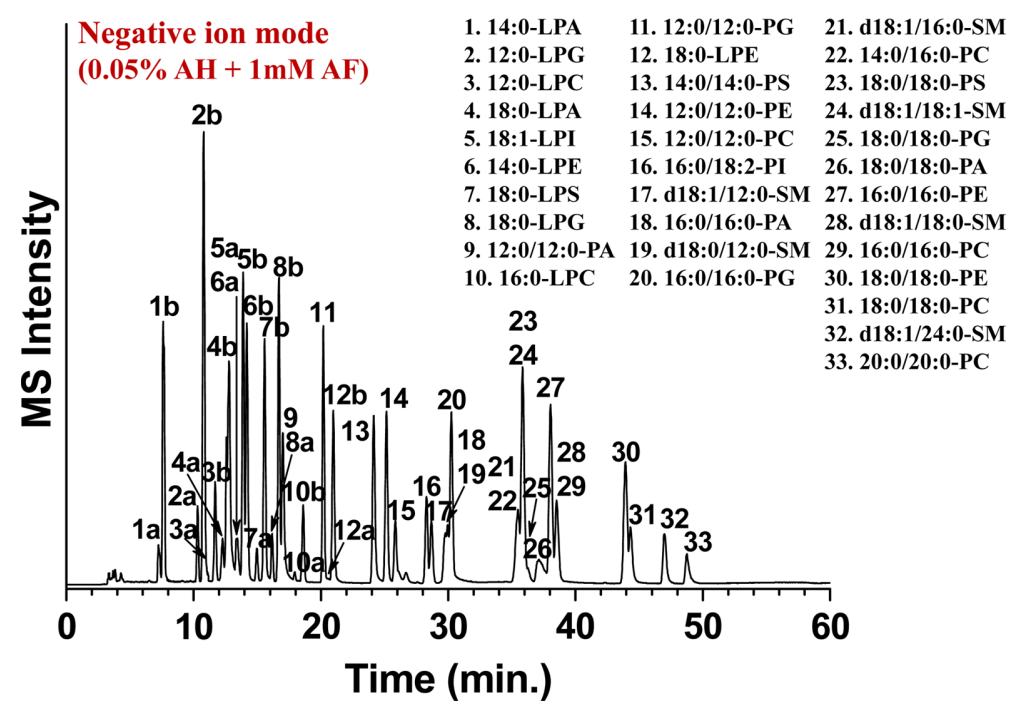

Figure 6. Base peak chromatograms of 33 PL standard mixtures from nLC-ESI-MS in negative ion mode. Peak numbers marked with a and $\mathrm{b}$ represent the regioisomers. Permission to reproduce obtained from the publisher of ref. 32 .
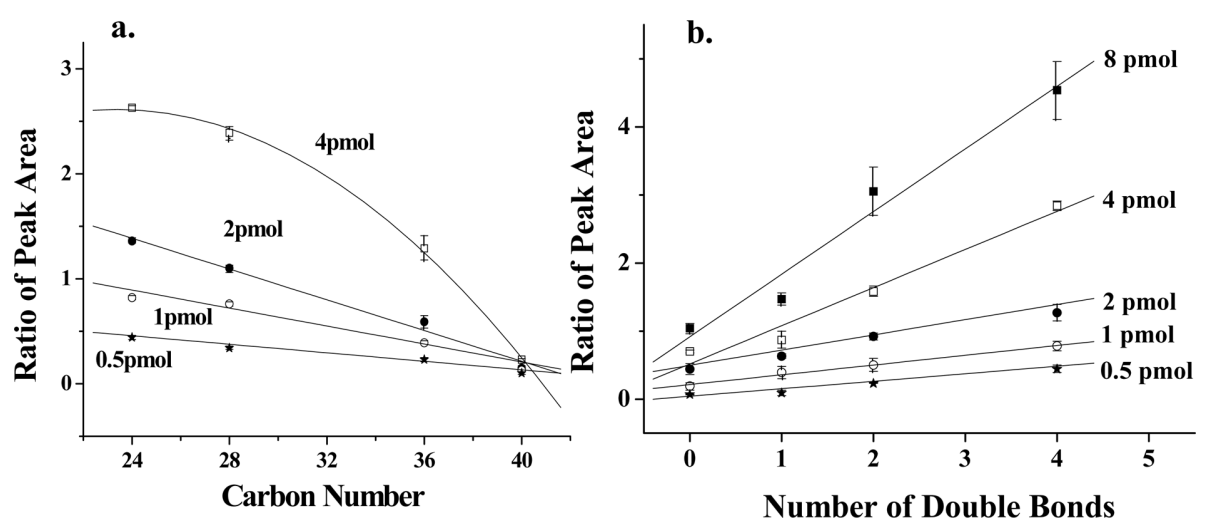

Figure 7. Effects of a) carbon number and b) unsaturation of acyl chain on MS peak intensity (based on relative peak area vs. IS) of PC standards according to injection amount. Permission to reproduce obtained from the publisher of ref. 26.

85 PL species identical to those obtained from a positive run and a negative run.

\subsection{Quantitative analysis of PLs by nLC-ESI-MS/MS}

PLs can be quantified by calculating the relative ion intensity of the PL molecule compared to those of single or multiple internal standards (IS), or by calculating the peak area from neutral loss scan. ${ }^{33,34}$ Because ionization of PLs depends strongly on the numbers of carbon and double bonds, however, it is often difficult to determine the concentration of PLs. It is known that ionization of lipids with shorter acyl chains is more favorable than those with longer chain due to differences in surface activity. ${ }^{35}$ In addition, double bonds in unsaturated acyl chains could weaken intermolecular interactions at the droplet surface, enhancing evaporation of the droplet during ESI. As shown in Figure $7 \mathrm{a}$, relative peak area (vs. IS) of PC standards obtained from nLC-ESI-MS decreases as the total carbon number of acyl chains increases, and the dependence of chain length decreases with the amount of individual species down to $0.5 \mathrm{pmol}$ for each species. In this case, the limit of detection (LOD) was calculated to be $2.22 \pm 0.33$ fmol $(n=9)$ based on $\mathrm{S} / \mathrm{N}=3$. Effect of unsaturation in the acyl chain on the relative peak area (vs. IS) of PC standards with acyl chain structures of 18:0/18:0, 18:0/18:1, 18:0/ 18:2, and 18:2/18:2 according to the amount of PC species injected is shown in Figure $7 \mathrm{~b}$. The larger the injection amount $(>2$ pmol) of each species, the larger the relative peak area due to the increase in number of double bonds. However, injection of $0.5 \mathrm{pmol}$ decreased the dependence on double bonds dramatically, as shown in Figure 7a, and the slope value of the calibration curve was 0.042 . This indicates that the effect of unsaturation of the acyl chain is less significant when the injection amount is maintained below 1 pmol. An earlier study ${ }^{26}$ demonstrated that the calibration curve for the calculation of PC concentrations 
can be obtained separately for each carbon length (24 40) as long as the amount of individual species is maintained at less than 1 pmol. Based on this, it was demonstrated that three-dimensional (3D) calibration curves can be established for each PL head group, at least for three standards with the same head group but different chain lengths. This was applied to determine the amounts of PCs and PEs from rat liver samples in a steatosis/fibrosis model $^{36}$ and to quantify human urinary PLs (a total 67) from patients with breast cancer. ${ }^{37}$ To profile relative changes in targeted PL species among different samples, the $3 \mathrm{D}$ calibration method is useful to determine absolute changes in amounts of PLs. However, in this case, at least three different PL standards of a head group by varying concentrations must be spiked into the samples to be analyzed, which yields data that is laborious to analyze.

For simple quantification of PLs, measurements of relative peak intensity (more likely peak area) of target PL molecules vs. IS can be taken when the relative change of a certain species needs to be determined among samples of different disease status in comparison to control samples. Peak area measurements of target PLs can be obtained directly from precursor ions or from the selected reaction monitoring method (SRM) once target molecular ions and their characteristic fragment ions are identified by CID experiments. The latter method yields more reproducible results than the former method when the complexity of the lipid sample is greater.

\section{Analysis of PLs from tissue and urine samples}

Feasibility of nLC-ESI-MS/MS for PL analysis was demonstrated with PC extracts ${ }^{24}$ from soybean, bovine brain, and liver, resulting in the identification of 28,25 , and 39 PCs and simultaneous characterization of their molecular structures by data-dependent CID experiments. nLC-ESI-MS/MS was then applied to liver tissue samples for quantitative profiling of PCs and PEs. PL extracts were obtained from a rat liver steatosis/fibrosis model induced by chronic administration of carbon tetrachloride to rats for 30 , 60 , and 90 days. By utilizing the 3D calibration of $\mathrm{PC}$ and PE with multiple standard addition and an internal standard, 37 PCs and 19 PEs identified by nLC-ESI-MS/MS were quantitatively profiled, demonstrating that relative regulation of individual PC and PE species can be traced under different physiologic and pathologic cellular states. Total PC and PE concentrations decreased by $\sim 51$ and $\sim 34 \%$, respectively, during the development of cirrhosis. ${ }^{26,36}$

When nLC-ESI-MS/MS was applied to analyze urinary phospholipids, it was found that it was challenging to extract PLs successfully from small volume urine samples. While human urine samples are generally large volume samples, sample volumes from animal models are usually limited. Therefore, an efficient extraction method to extract PLs successfully from small volume urine samples needs to be developed. In a preliminary study, ${ }^{38} 75$ PLs from six different PL categories were successfully identified from only $1 \mathrm{~mL}$ of human urine by adopting a lyophilization step rather than ultracentrifugation to remove water from the urine prior to use of the Folch method. Quantitative analysis of human urinary PLs from breast cancer (BC) patients and controls using the $3 \mathrm{D}$ calibration method resulted in structural identification of a total of $55 \mathrm{PLs} ;{ }^{37}$ the majority of PC and PE species that showed a significant increase in patients had 16:0 or 18:0 acyl chains at the sn-1 position and 18:1 or 18:2 chains at the sn- 2 position. These findings are similar to a literature report ${ }^{39}$ in which the StarD10 protein, known to be overexpressed in BC, preferentially captured lipids species with 16:0 (palmitoyl) and 18:0 (stearoly) chains at the sn-1 position and $18: 1$ or $18: 2$ chains at the sn-2 position. Studies also demonstrated that the total concentration of PCs and PEs in $\mathrm{BC}$ patients increased by 44 and $71 \%$, respectively, compared to control samples but decreased significantly by 20 and $78 \%$ after surgery, respectively.

\section{Analysis of PLs in human plasma}

\subsection{PLs in plasma lipoproteins by flow field-flow frac- tionation and $n L C-E S I-M S / M S$}

Phospholipid analysis of plasma sample with nLC-ESIMS/MS can be carried out in two different ways: by extracting PLs from whole plasma ${ }^{31}$ using extraction methods described in Section 2.1 or by extracting PLs from different lipoprotein subclasses preliminary fractionated by size using flow field-flow fractionation (FIFFF). ${ }^{14,40}$ Lipoproteins are globular particles in blood that contain neutral lipids such as triglycerides (TG), diglycerides (DG), and cholesteryl esters (CE) in the core of the particle and polar lipids like PLs with some proteins in the exterior; their roles are to transfer fats and cholesterol through the body. Lipoproteins are classified by density as high density lipoprotein (HDL), low density lipoprotein (LDL), and very low density lipoprotein (VLDL), and their sizes increase with a decrease in density. Because low levels of HDL and high levels of LDL with a size reduction of LDL are known to be strongly related to coronary artery disease (CAD), ${ }^{41,42}$ elucidating the different patterns of lipids from HDL and LDL fractions may help in clarifying the involvement of lipid metabolism in cardiovascular disease. By utilizing FlFFF, HDL and LDL particles can be size-sorted and separately collected for the further analysis in both qualitative and quantitative purposes after lipid extraction. Figure 8a shows the multiplexed hollow fiber FIFFF (mHF5) channel assembly ${ }^{40}$ developed for semi-preparative scale separation of HDL/LDL from plasma. Six hollow fiber channels are connected in parallel to increase the injection amount (up to $50 \mu \mathrm{L}$ per injection), and the fractogram shown in the top of panel $b$ demonstrates the baseline separation of HDL and LDL particles along with standard protein separation at the bottom. ${ }^{14}$ HDL/LDL fractions 

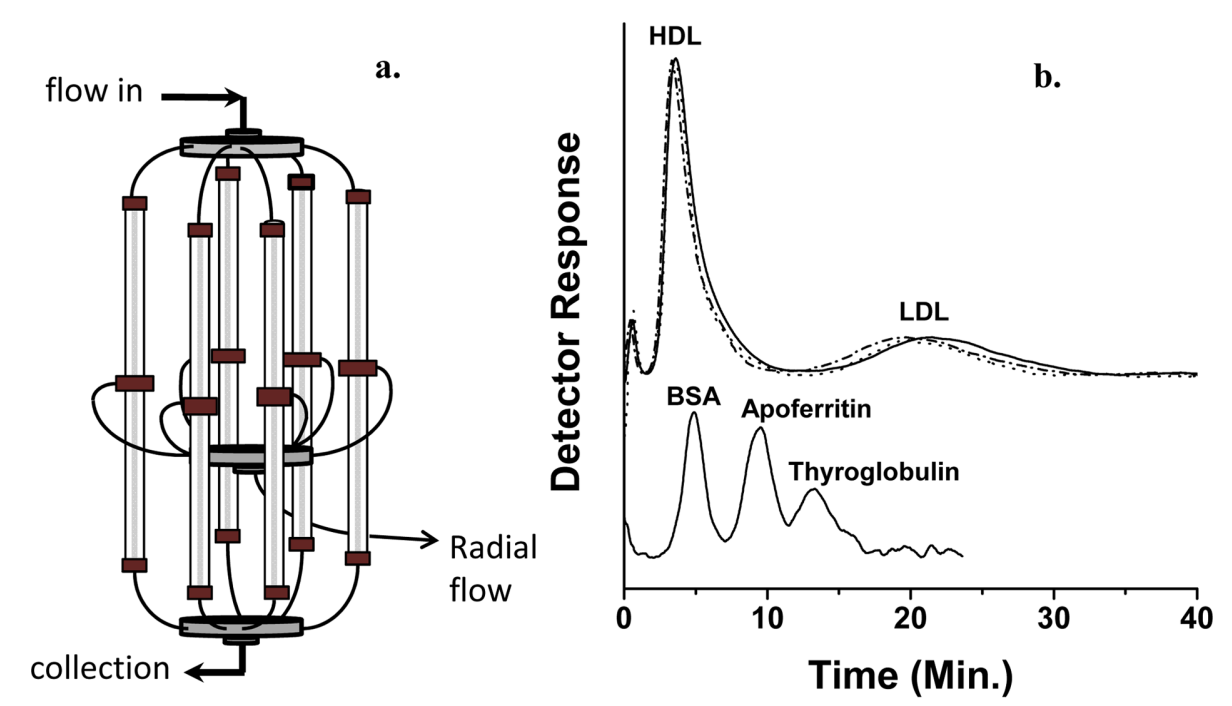

Hollow Fiber modules

Figure 8. a) Configuration of multiplexed hollow fiber flow field-flow fractionation (MxHF5) and b) fractograms of MxHF5 separation of HDL and LDL particles from $50 \mu \mathrm{L}$ of human plasma (stained with SBB) detected at $600 \mathrm{~nm}$ (up), and b) three proteins standards $\left(15 \mu \mathrm{g}\right.$ each) at $280 \mathrm{~nm}$ (bottom). For both runs, the flow rate condition was $\dot{V}_{\text {out }} / \dot{V}_{\text {rad }}=0.8 / 2.2 \mathrm{~mL} / \mathrm{min}$. Panel b reproduced with permission from the publisher of ref. 14.

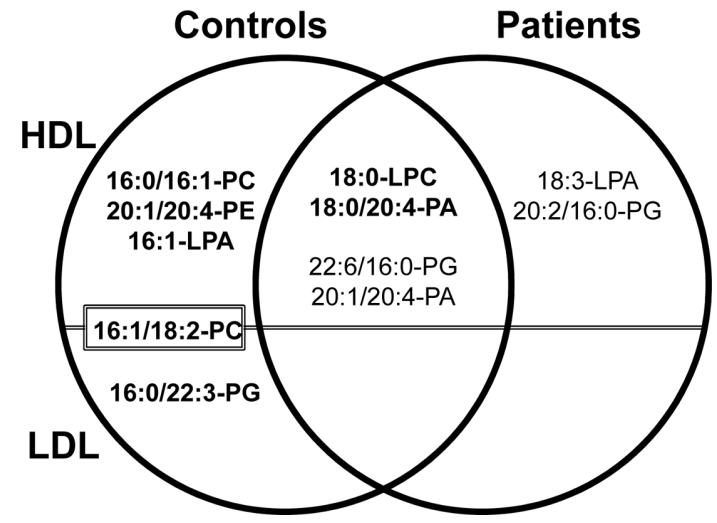

Figure 9. Candidate PL and LPL biomarkers found exclusively in control (five species in the left circle) and patient groups (two in the right circle). Molecules marked with bold in the overlapping region (center) indicate those that showed a significant decrease $(>$ 5-fold) in patients, while those in plain text denote those molecules that showed a significant increase in patients. Permission to reproduce obtained from the publisher of ref. 14 .

were collected for lipid extraction followed by nLC-ESIMS/MS analysis, resulting in identification of 93 PLs. Among these species, quantitative analysis with statistical treatment yielded 11 PL species that showed significant changes ( $>5$ fold) in their relative amounts; these are shown in Figure 9. For example, three species (16:0/16:1PC, 20:1/20:4-PE, and 16:1-LPA) were found exclusively found in HDL from control subjects, while 18:3-LPA and 20:2/16:0-PG were present only in LDL from patients; these are therefore good candidate CAD markers.

\subsection{Characterization of oxidized PLs in oxidized LDLs}

A recent study characterized oxidized LDLs (Ox-LDLs), defined as oxidatively modified LDLs, at the molecular level using nLC-ESI-MS ${ }^{\mathrm{n}}{ }^{43}$ Oxidation of LDLs occurs primarily at the unsaturated acyl chain of PLs, resulting in the production of short chain products and long chain products. Oxidized PLs (Ox-PLs) are known to induce physical changes in biological membranes and to be involved in the formation of plaques in the inner walls of blood vessel, leading to atherosclerosis. ${ }^{44,45}$ To understand the patterns of Ox-LDL at the molecular level, bilayer vesicles prepared with a single PL standard were oxidized with a $\mathrm{Cu}^{2+}$ solution first, and the resulting Ox-PL products were systematically examined with nLC-ESI-MS ${ }^{\mathrm{n}}$. Figure 10 shows the separation of a) a mixture of 8 PL standards and b) Ox-PA products from the oxidation of bilayer vesicles prepared with standard 18:0/22:6-PA species (peak \#8 in panel a) along with c-g) CID spectra of Ox-PA products. ${ }^{43}$ Figure $10 \mathrm{~b}$ shows numerous Ox-PA products that eluted between $15-30 \mathrm{~min}$, indicating that oxidized species have a shorter retention time due to addition of hydroxide or hydroperoxide, or lack of an acyl chain. Each of the numbered species in Figure 10b was examined by CID spectra. Figure 10c shows the CID spectra of the intact 18:0/22:6-PA species. Typical fragment ions including free carboxylate anions (fragments (1) and (2)) and loss of acyl chains in the form of carboxylic acid (fragments (5) and (3)) or ketene (fragments (6) and (4)) from both sn-1 and sn-2 chains, respectively, were observed. Fragment ions with varied $\mathrm{m} / \mathrm{z}$ values due to the shortness of the $\mathrm{sn}-2$ chain or oxygen addition to the sn- 2 chain were observed in 


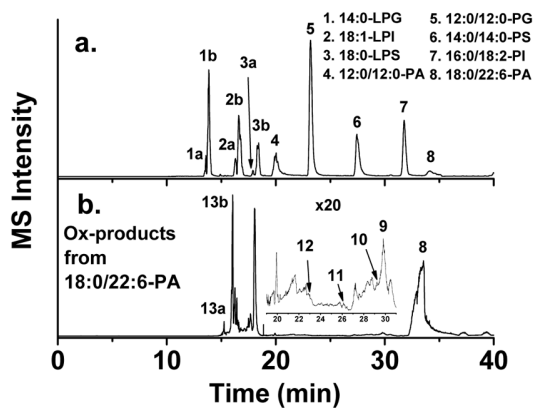

d. CID of \#10 (779.6, $29.6 \mathrm{~min})$ 18:0/22:6(+OO)-PA

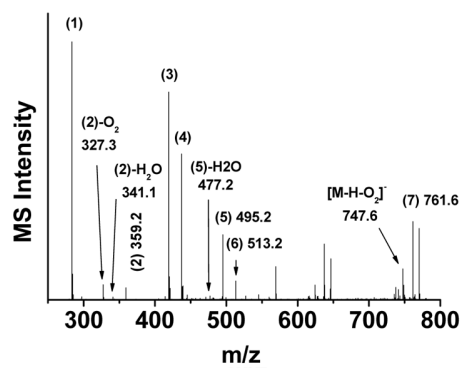

e. CID of \#12 (737.6, $23.3 \mathrm{~min})$ 18:0/19:5(COOH)-PA

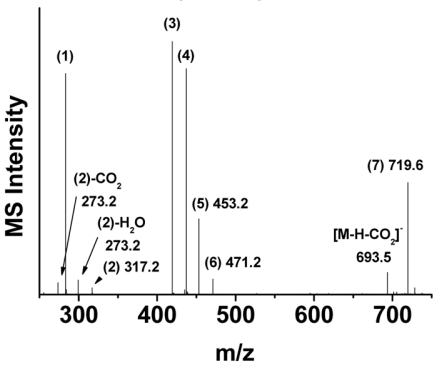

C. CID of \#8(747.6,34.0 min)

18:0/22:6-PA

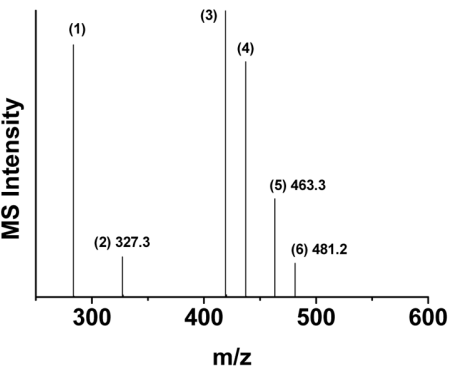

f. CID of \#13a (437.1, 15.2 min) lyso/18:0-PA

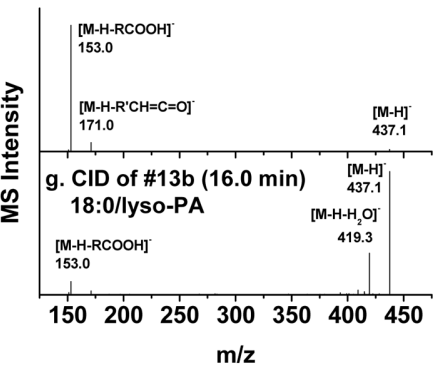

Figure 10. Base peak chromatograms (BPCs) of a) eight PL standards, b) Ox-PA species obtained from oxidative modification of 18:0/ 22:6-PA standards (peak \#18), data-dependent CID spectra of Ox-PA species: c) peak \#8, d) peak \#10, e) peak \#12, f) peak \#13a and g) peak \#13b. Spectra c)-e) show the same fragment ions as (1) $\left[\mathrm{R}_{1} \mathrm{COO}\right]^{-}, \mathrm{m} / \mathrm{z} 283.4$, (3) $\left[\mathrm{M}-\mathrm{H}-\mathrm{R}_{2} \mathrm{COOH}\right]^{-}, \mathrm{m} / \mathrm{z} 419.2$, and (4) [M-H$\left.\mathrm{R}_{2}{ }^{\prime} \mathrm{CH}=\mathrm{C}=\mathrm{O}\right]^{-}, \mathrm{m} / \mathrm{z}$ 437.3. The same type of fragment ions with varied $\mathrm{m} / \mathrm{z}$ values for each spectra are $(2)\left[\mathrm{R}_{2} \mathrm{COO}\right]^{-},(5)[\mathrm{M}-\mathrm{H}-$ $\left.\mathrm{R}_{1} \mathrm{COOH}\right]^{-}$, (6) [M-H- $\left.\mathrm{R}_{1}{ }^{\prime} \mathrm{CH}=\mathrm{C}=\mathrm{O}\right]^{-}$, and (7) $\left[\mathrm{M}-\mathrm{H}-\mathrm{H}_{2} \mathrm{O}\right]^{-}$. Permission to reproduce obtained from the publisher of ref. 43.

the CID spectra of Ox-PA products. Figure 10d shows additional fragments resulting from dissociation of $\mathrm{O}_{2}$ from the parent ion $\left[\mathrm{M}-\mathrm{H}-\mathrm{O}_{2}\right]^{-}$at $m / z 747.6$ and from the free carboxylate ion as [(2)- $\left.\mathrm{O}_{2}\right]^{-}$at $m / z 327.3$, as well as several fragments produced by the loss of water from the sn-2 acyl chain, which supports the addition of two oxygens in the form of a peroxide (different from the two hydroxide addition). Figure 10e shows the loss of $\mathrm{CO}_{2}$ from the free carboxylate anion at sn-2 and from the parent molecule, indicating that the sn-2 acyl chain was terminated by a carboxylic acid after truncation of the acyl chain into 18:0/ 19:5(COOH)-PA. Noteworthy is the formation of two regioisomers of 18:0-LPA during oxidative dissociation of the sn-2 acyl chain, as shown by the distinct separation of peaks 13a and 13b in Figure 10b and the characteristic CID spectra in Figures $10 \mathrm{f}$ and $10 \mathrm{~g}$; the ratio of [M-H-RCOOH $]^{-}$ $/[\mathrm{M}-\mathrm{H}]^{-}$for the two regioisomers was reversed. This indicates that the specific location of an acyl chain of LPL can be exchanged between the sn- 1 and sn-2 positions during oxidative dissociation of the acyl chain. A number of different forms of Ox-PL products have been characterized. ${ }^{43}$ An oxidation method was applied to standard LDL; Ox-PLs from oxidized LDL were analyzed with nLC-ESI-MS ${ }^{\mathrm{n}}$, resulting in identification of a total of 276 PLs, including 139 Ox-PL species. Molecular patterns obtained from systematic analysis provide a good basis with which to characterize Ox-
PLs from human plasma, and studies are on-going to quantitatively profile Ox-PLs of LDL particles from human plasma samples of CAD patients.

\section{Conclusions}

In the present review, described are the merits of nLCESI-MS/MS for the separation and characterization of lipid mixtures from various biological sources, and noted are some experimental necessities that should be considered for successful analysis. Because lipids have complicated and diverse electrical propensities, it is difficult to establish a universal extraction method for simultaneous retrieval of all kinds of lipids from biological samples or design a universal analytical platform for simultaneous analysis of different categories of lipids. Recent advancements in MS have facilitated the accurate determination of mass values of lipid molecules along with sound information on their molecular structures; however, sample complexity still needs to be reduced using an on-line separation method to enable successful detection of each species without biasing quantitative information. Nanoflow LC separation ( $\sim 300 \mathrm{~nL} / \mathrm{min})$ prior to ESI-MS/MS has advantages for lipid analysis. Detection limit can be lowered to the low fmol level, and low abundance lipid species can be detected better because ion suppression can be substantially reduced 
by resolution enhancement in a microscale separation system. In addition, the amount of lipids injected can be minimized, because all the LC effluent is infused directly to the ESI without splitting flow and without using a sheath gas. Minimization of the amount of lipid injected is important for MS detection of lipid species, because the influence of the unsaturation degree of the acyl chain on MS intensity is negligible at below 1 pmol. However, separation still needs to be improved due to the complexity of the lipidome. Efforts to improve separation that involve multidimensional separation, such as normal phase LC $(\mathrm{NPLC})^{46}$ or strong anion exchange $(\mathrm{SAX})^{47}$ in the first dimension connected online to RPLC in the second dimension to enhance lipid identification are made recently. However, a comprehensive separation method to improve lipid analysis is required, as are systematic developments in the bioinformatics of lipids.

\section{Acknowledgements}

This study was supported by a grant (NRF-20100014046) from the National Research Foundation (NRF) of Korea funded by the Korean government (MEST).

\section{References}

1. Miekle, P. J.; Christopher, M. J. Curr. Opin. Lipidol. 2011, 22, 210.

2. Brouwers, J. F. H. M.; Vernooji, E. A. A. M.; Tielens, A. G. M.; van Golde, L. M. G. J. Lipid Res. 1999, 40, 164.

3. Wright, M. M.; Howe, A. G.; Zaremberg, V. Biochem. Cell Biol. 2004, 82, 18.

4. Houjou, T.; Yamatani, K.; Imagawa, M.; Shimizu, T.; Taguchi, R. Rapid Commun. Mass Spectrom. 2005, 19, 654.

5. Bougnoux, P.; Chajes, V.; Lanson, M.; Hacene, K.; Body, G.; Couet, C.; Folch, O. L. Breast Cancer Res. Treat. 1992, 20, 185.

6. Leach, M. O.; Verrill, M.; Glaholm, J.; Smith, T. A. D.; Collins, D. J.; Payne, G. S.; Sharp, J. C.; Ronen, S. M.; McCready, V. R.; Powles, T. J.; Smith, I. E. NMR Biomed. 1998, $11,314$.

7. Wolf, C.; Quinn, P. J. Prog. Lipid Res. 2008, 47, 15.

8. Kim, H.; Min, H. K.; Kong, G.; Moon, M. H. Anal. Bioanal. Chem. 2009, 393, 1649.

9. Xu, Y.; Shen, Z.; Wiper, D. W.; Wu, M.; Morton, R. E.; Elson, P.; Kennedy, A. W.; Belinson, J.; Markman, M.; Casey, G. JAMA. 1998, 280, 719.

10. Sutphen, R.; Xu, Y.; Wilbanks, G. D.; Fiorica, J.; Grendys Jr., E. C.; LaPolla, J. P.; Arango, H.; Hoffman, M. S.; Martino, M.; Wakeley, K.; Griffin, D.; Blanco, R. W.; Cantor, A. B.; Xiao, Y. J.; Krischer, J. P. Cancer Epidemiol. Biomarkers Prev. 2004, 13, 1185.

11. Zhao, Z.; Xiao, Y.; Elson, P.; Tan, H.; Plummer, S. J.; Berk, M.; Aung, P. P.; Lavery, I. C.; Achkar, J. P.; Li, L.;
Casey, G.; Xu, Y. J. Clin. Oncol. 2007, 25, 2696.

12. Swanson, M. G.; Vigneron, D. B.; Tabatabai, Z. L.; Males, R. G.; Schmitt, L.; Carroll, P. R.; James, J. K.; Hurd, R. E.; Kurhanewicz, J. Magn. Reson. Med. 2003, $50,944$.

13. Jeong, R. U.; Lim, S.; Kim, M. O.; Moon, M. H. Anal. Bioanal. Chem. 2011, 401, 689.

14. Byeon, S. K.; Lee, J. Y.; Lim, S.; Choi, D.; Moon, M. H. J. Chromatogr. A 2012, 1270, 246.

15. Helmy, F. M.; Nosavanh, L.; Haynes, H.; Juracka, A. Cell Biochem. Funct. 2008, 26, 434.

16. Murray, K. E.; Fried, B.; Sherma, J. Acta Chromatogr. 2007, 18, 190.

17. Fuchs, B.; Schiller, J.; Sü $\beta$, R.; Schürenberg, M.; Suckau, D. Anal. Bioanal. Chem. 2007, 389, 827.

18. Rohlfing, A.; Muthing, J.; Pojlentz, G.; Distler, I.; PeterKatalinc, J.; Berkenkamp, S.; Dreisewerd, K. Anal. Chem. 2007, 79, 5793.

19. Taguchi, R.; Hayakawa, J.; Takeuchi, Y.; Ishida, M. J. Mass Spectom. 2000, 35, 953.

20. Isaac, G.; Bylund, D.; Mansson, J. -E.; Markides, K. E.; Borgquist, J. J. Neurosci. Methods 2003, 128, 111.

21. Hermansson, M.; Uphoff, A.; Kakela, R.; Somerharju, P. Anal. Chem. 2005, 77, 2166.

22. Oursel, D.; Loutelier-Bourhis, C.; Orange, D.; Chevalier, S.; Norris, V.; Lange, C. M. Rapid Commun. Mass Spectrom. 2007, 21, 1721.

23. Pacetti, D.; Borselli, E.; Lucci, P.; Frega, N. G. J. Chromatogr. A 2007, 1150, 242.

24. Bang, D. Y.; Kang, D.; Moon, M. H. J. Chromatogr. A 2006, 1104, 222.

25. Bang, D. Y.; Ahn, E. J.; Moon, M. H. J. Chromatogr. B 2007, 852, 268.

26. Ahn, E. J.; Kim, H.; Chung, B. C.; Moon, M. H. J. Sep. Sci. 2007, 30, 2598.

27. Byeon, S. K.; Lee, J. Y.; Moon, M. H. Analyst 2012, 137, 451.

28. Folch, J.; Lees, M.; Sloan Stanley, G. H. J. Biol. Chem. 1957, 226, 497.

29. Bligh, E. G.; Dyer, W. J. Can. J. Biochem. Physiol. 1959, 37, 911.

30. Lim, S.; Byeon, S. K.; Lee, J. Y.; Moon, M. H. J. Mass Spectrom. 2012, 47, 1004.

31. Lee, J. Y.; Min, H. K., Moon, M. H. Anal Bioanal. Chem. 2011, 400, 2953.

32. Bang, D. Y.; Lim, S. Moon, M. H. J. Chromatogr. A 2012, 1240, 69.

33. Fang, J.; Barcelona, M. J. J. Microbiol. Methods 1998, 33, 23.

34. Taguchi, R.; Houjou, T,; Nakanishi. H.; Yamazaki, T.; Ishida, M.; Imagawa, M.; Shimizu, T. J. Chromatogr. B 2005, 823, 26.

35. Koivusalo, M.; Haimi, P.; Heikinheimo, L,; Kostiainen, R.; Somerharaju, P. J. Lipid Res. 2001, 42, 663.

36. Ahn, E. J.; Kim, H.; Chung, B. C.; Kong, G.; Moon, M. H. J. Chromatogr. A 2008, 1194, 96. 
37. Min, H. K.; Kong, G.; Moon, M. H. Anal. Bioanal. Chem. 2010, 396, 1273.

38. Kim, H.; Ahn, E. J.; Moon, M. H. Analyst 2008, 133, 1656.

39. Olayioye, M.; Vehring, S.; Muller, P.; Herrmann, A.; Schiller, J.; Thiele, C.; Linderman, G. J.; Visvader, J. E.; Pomorski, T. J. Biol. Chem. 2005, 280, 27476.

40. Lee, J. Y.; Min, H. K.; Choi, D.; Moon, M. H. J. Chromatogr. A 2010, 1217, 1660.

41. Rajman, I.; Kendall, M. J.; Cramb, R.; Holder, R. L.; Salih, M.; Gammage, M. D. Atherosclerosis 1996, 125, 231.

42. Adler, L.; Hill, J. S.; Frohlich, J. Clin. Biochem. 2000, 33, 187.
43. Lee, J. Y.; Lim, S.; Park, S.; Moon, M. H. J. Chromatogr. $A$ 2013, 1288, 54.

44. Ehara, S.; Ueda, M.; Naruko, T.; Haze, K.; Itoh, A.; Otsuka, M.; Komatsu, R.; Matsuo, T.; Itabe, H.; Takano, T.; Tsukamoto, Y.; Yoshiyama, Y.; Takeuchi, K.; Yoshikawa, J.; Becker, A. E. Circulation 2001, 103, 1955.

45. Adachi, J.; Asano, M.; Yoshioka, N.; Nushida, H.; Ueno, Y. Kobe J. Med. Sci. 2006, 52, 127.

46. Nie, H.; Liu, R.; Yang, Y.; Bai, Y.; Guan, Y.; Qian, D.; Wang, T.; Liu, H. J. Lipid Res. 2010, 51, 2833.

47. Bang, D. Y.; Moon, M. H. J. Chromatogr. A 2013, 1310, 82 . 